\title{
La semaine motivationnelle : une nouvelle approche dans le sevrage tabagique
}

\author{
L. Hanssens ${ }^{\mathrm{a},}{ }^{,}$, V. Lustygierb, M. Ansseau ${ }^{b}$, I. Thiebauta, J . Thimpontc \\ aUnité de tabacologie, service de pneumologie, hôpital universitaire des enfants Reine Fabiola, université \\ Libre de Bruxelles (ULB), 15, avenue J.J.-Crocq, 1020 Bruxelles, Belgique \\ ${ }^{b}$ Unité de tabacologie, service de psychiatrie, CHU Brugmann, université Libre de Bruxelles (ULB), \\ Bruxelles, Belgique \\ 'Unité de tabacologie, service de pneumologie, CHU Brugmann, université Libre de Bruxelles (ULB), \\ Bruxelles, Belgique
}

KEYWORDS: tabagisme, sevrage tabagisme, motivation, dépression, thérapeutique

\section{ABSTRACT}

Introduction. - Le sevrage tabagique est complexe et difficile. La semaine motivationnelle est une approche pluridisciplinaire qui a été instaurée afin d'augmenter les chances d'arrêt du fumeur. Le but de cette étude était d'en déterminer les taux d'abstinence et les facteurs prédictifs de l'arrêt.

Méthodes. - Les données cliniques, le statut tabagique, le degré de dépendance, de motivation et les taux d'abstinence continue à court et à long terme de tous les patients qui ont participé à une semaine motivationnelle ont été analysés.

Résultats. - Deux cent treize patients ont été inclus. L'âge moyen était de 49,8 $\pm 10,6$ ans. Les taux d'abstinence continue étaient de 40,4\%,29,1\% et 21,6\% respectivement à 6 mois, 12 mois et 2 ans. En régression logistique, une dépression ou des antécédents de dépression étaient associés à une probabilité plus faible d'abstinence tabagique : ORa : 0,32 [IC 95 \%:0,16-0,76; $p$ $=0,003$ ] à 6 mois, ORa :0,35 [IC $95 \%: 0,16-0,77 ; p=0,009$ ] à 1 an et ORa : 0,27 [IC $95 \%: 0$, $11-0,65 ; p=0,004]$ à 2 ans.

Conclusions. - La semaine motivationnelle semble être une approche efficace à long terme qui pourrait être utilisée dans le sevrage tabagique. Cette étude confirme que la dépression est un facteur défavorable de l'arrêt de l'intoxication tabagique. 


\section{Introduction}

Le tabagisme reste la principale cause évitable de morbidité et de mortalité dans le monde. L'arrêt de la consommation du tabac offre des bénéfices tant pour l'individu lui-même que pour son entourage [1-4]. Le tabac est une drogue dure par la triple dépendance qu'il induit (physique, psychologique et comportementale). Le sevrage tabagique est donc complexe et difficile. En effet, même si 2 à $5 \%$ des fumeurs décidés à arrêter réussissent à le faire sans intervention [5], et même si les différents traitements à notre disposition permettent d'améliorer de manière significative les taux d'abstinence, le sevrage tabagique reste associé à un taux élevé de rechutes. La prise en charge du patient fumeur doit, donc, être globale.

La semaine motivationnelle est une prise en charge pluridisciplinaire, développée dans le but d'augmenter les chances d'abstinence du patient tabagique. Elle fait partie, depuis juillet 2003, de l'aide au sevrage des patients suivis dans notre institution hospitalière. Elle est proposée à tous les patients favorables à une approche en groupe. La prise en charge se fait en hôpital de jour durant une semaine ( 5 jours ouvrables) par une équipe pluridisciplinaire com prenant un psychiatre tabacologue, un pneumologue tabacologue, des psychologues tabacologues, un kinésithérapeute et une diététicienne. L'arrêt du tabac est proposé aux patients à partir du troisième jour de la semaine. La semaine motivationnelle est basée sur une approche psychologique, motivationnelle, individuelle et de groupe. Elle comporte des informations sur les conséquences du tabagisme et sur le mécanisme bio-psychosocial de l'assuétude, la pratique du sport, l'hypnose, la gestion du stress par l'apprentissage de techniques de relaxation, des conseils diététiques et des jeux de rôle. Elle permet ainsi une prise en charge globale du patient. Afin de limiter les risques de rechutes, les patients sont suivis jusqu'à un an après l'arrêt.

L'objectif principal de cette étude consistait à évaluer l'efficacité de la semaine motivationnelle par le calcul des taux d'abstinence continue à court et à long terme. L'objectif secondaire consistait à identifier les facteurs prédictifs de l'arrêt par l'étude des variables associées à l'abstinence.

\section{Matériel et méthodes}

Cette étude était rétrospective. Les données ont été obtenues à partir du dossier de consultation tabagique (validé par la Société française de tabacologie ; www.inpes.sante.fr) de tous les patients qui ont participé entre le 1er juillet 2003 et le 30 juin 2010 à la semaine motivationnelle du centre hospitalier universitaire Brugmann à Bruxelles (Belgique). Les données suivantes ont été recueillies : l'âge, le sexe, les comorbidités (hypertension artérielle, hypercholestérolémie, diabète, infarctus du myocarde, accident vasculaire cérébral, artérite, bronchopneumopathie chronique obstructive, asthme, cancer, dépression), la co-dépendance à l'alcool évaluée par le test DETA-CAGE (probabilité élevée d'une alcoolo-dépendance si 2 réponses positives) [6], la durée du tabagisme, la consommation journalière de tabac, les antécédents de sevrage défini par un arrêt antérieur de plus de 7 jours, la dépendance physique évaluée par le test de Fagerstrëm [7], la motivation évaluée par le test de Richmond [8] et par une échelle analogique de 0 à 10 
(motivation définie comme bonne si score $\geq 8$ ) ainsi que l'état anxieux ou dépressif identifié par le test Hospital Anxiety Depression (HAD) [9].

Les patients qui n'ont pas terminé la semaine motivationnelle ou qui y avaient déjà participé ou qui étaient atteints d'une pathologie psychiatrique sévère (schizophrénie, troubles bipolaires, suicides, toxicomanie, éthylisme sévère...) ou qui ont combiné une autre forme de thérapie de sevrage tabagique ont été exclus de l'analyse.

\section{TAUX D'ABSTINENCE}

Les taux d'abstinence continue (aucun Lisage du tabac depuis l'arrêt) ont été calculés à 6 mois, 1 an et 2 ans. Ces résultats ont été obtenus sur base des déclarations des patients, 6 mois et 1 an après leur participation à la semaine motivationnelle, lors du suivi en consultation ou par contact téléphonique. Tous les patients ont également été contactés par téléphone après 2 ans. Les perdus de vue ont été considérés comme des fumeurs persistants.

L'étude a reçu un avis favorable du comité d'éthique du centre hospitalier universitaire Brugmann.

\section{STATISTIQUES}

La normalité des variables quantitatives a été étudiée à l'aide du plot normal. Les statistiques descriptives usuelles ont été utilisées : moyenne et déviation standard pour les variables normales, médiane et étendue (minimum et maximum) pour les variables asymétriques et pourcentages (\%) pour les variables en catégories. Afin de déterminer les facteurs prédictifs de l'arrêt, une analyse univariée (OR) et multivariée (ORa) par régression logistique pour tenir compte des effets potentiellement confondants a été utilisée. La sélection des prédicteurs dans le modèle a été effectuée par une méthode de sélection progressive basée sur le rapport de vraisemblance. Seuls les effets statistiquement significatifs $(\mathrm{p}<0,05)$ ont été retenus dans le modèle final. De ce modèle, des OR ajustés (ORa) et leurs index de confiance à $95 \%$ (IC $95 \%$ ) ont été dérivés, le test de Wald a été appliqué. Les analyses ont été réalisées à l'aide du logiciel STATA, version 10.1 et le seuil de signification statistique retenu était de $5 \%$.

\section{RÉSULTATS}

Deux cent soixante-quatre personnes ont participé à la semaine motivationnelle. Cinquante et un patients ont été exclus : 3 n'ont pas terminé la semaine motivationnelle, 3 y avaient déjà participé, 24 étaient atteints d'une pathologie psychiatrique sévère et 21 ont combiné un traitement médicamenteux. Au total, 213 patients ont été inclus dans l'étude.

En raison des perdus de vue, le nombre total de patients inclus aux différentes périodes étudiées était de 165 à 6 mois, de 162 à un an et de 161 à 2 ans.

\section{CARACTÉRISTIQUES DE LA POPULATION}

L'âge moyen des patients était de 49,8 \pm 10,6 ans (Tableau 1). Parmi les comorbidités les plus fréquentes, $40,4 \%$ patients étaient dépressifs ou avaient des antécédents de dépression et le 
test HAD révélait un état dépressif chez $27,1 \%$ des patients ; 23,3 \% étaient atteints de BPCO et 20,3\% avaient une hypertension artérielle systémique.

\section{TAUX D'ABSTINENCE CONTINU}

Sur les 213 patients initiaux, 86 patients étaient abstinents à 6 mois (40,4\%), 62 patients à 1 an $(29,1 \%)$ et 46 patients à 2 ans $(21,6 \%)$.

\begin{tabular}{|ll|}
\hline $\begin{array}{l}\text { Tableau } 1 \text { Caractéristiques de la population totale } \\
\text { d'étude. }\end{array}$ & $n=213$ \\
\hline & $49,8 \pm 10,6$ \\
\hline $\begin{array}{l}\text { Âge moyen (ans) } \\
\text { Sex ratio }(M / F)\end{array}$ & $83 / 130$ \\
$\begin{array}{l}\text { Dépression ou antécédents de } \\
\text { dépression, } n \text { (\%) }\end{array}$ & $86(40,4)$ \\
$\begin{array}{l}\text { Alcoolo-dépendance, } n(\%) \\
\text { Statut tabagique } \\
\text { Durée du tabagisme (années) } \\
\text { Consommation journalière } \\
\text { (nombre de cigarettes/jour) }\end{array}$ & $88(41,3)$ \\
$\begin{array}{ll}\text { Arrêt antérieur }>7 \text { jours, } n(\%) \\
\text { Dépendance physique }\end{array}$ & $26,3 \pm 10,5$ \\
$\begin{array}{l}\text { Test de Fagerström } \\
\text { Motivation a l'arrêt } \\
\text { Échelle de } 0 \text { à } 10 \text { (médiane) }\end{array}$ & $154(72,3)$ \\
Test de Richmond (médiane) & 8 (extrêmes : 2-10) \\
\hline
\end{tabular}

\section{FACTEURS PRÉDICTIFS DE L'ARRÊT}

L'analyse par régression logistique univariée (OR) et multivariée (ORa) est résumée dans le Tableau 2 et montre qu'à toutes les périodes étudiées, la dépression ou des antécédents de dépression étaient associés à une probabilité plus faible d'abstinence tabagique. En analyse univariée, la motivation évaluée par l'échelle analogique était associée à une probabilité plus élevée d'abstinence à toutes les périodes étudiées mais n'était plus significative, en analyse multivariée, à 2 ans.

\section{DISCUSSION}

La prise en charge du patient fumeur doit être globale. En effet, par la triple dépendance que le tabagisme induit, le sevrage tabagique est complexe et difficile. Quelle que soit la méthode d'arrêt utilisée, les taux d'abstinence res-tent faibles et les taux de rechutes élevés. La semaine motivationnelle est un projet original de prise en charge pluridisciplinaire du sevrage tabagique qui tient compte également de l'aspect évolutif du sevrage par le suivi régulier des patients jusqu'à un an après l'arrêt. Les objectifs de cette étude étaient d'évaluer l'efficacité de cette nouvelle approche et d'identifier les facteurs influençant la réussite du sevrage.

Si on compare les résultats d'abstinence ( $40,4 \%$ à 6 mois, $29,1 \%$ à 1 an et $21,6 \%$ à 2 ans) à ceux des autres approches thérapeutiques utilisées, particulièrement les approches non médicamenteuses, la semaine motivationnelle semble être une approche efficace. Dans la métaanalyse d'Agboola et al., les taux d'abstinence à 1 an étaient de $22 \%$ (IC $95 \%: 19-25 \% ; n=19$ ) pour la varénicline et de $8 \%$ (IC $95 \%: 6-12 \% ; n=19$ ) pour le placebo [10]. Fiore et al. ont montré que les taux d'abstinence à un an pour les approches médicamenteuses étaient de 24,2 
$\%$ pour le bupropion et variaient entre 19,0 et $26,5 \%$ pour les substituts nicotiniques selon les formes, les doses et les durées utilisées et entre $25,4 \%$ et 33,2 \% pour la varénicline en fonction de la dose utilisée. Pour les approches non médicamenteuses, les taux d'abstinence à un an n'étaient que de $12,3 \%$ pour le self help, de $13,9 \%$ pour le soutien de groupe et de 16,8 \% pour le soutien individuel [11]. Enfin, plus récemment, une revue Cochrane [12] a confirmé que les substituts nicotiniques, la varénicline et le bupropion étaient plus efficaces en termes d'abstinence que le placebo. L'association des substituts nicotiniques et la varénicline étaient plus efficaces que le bupropion ou l'utilisation d'un seul substitut nicotinique. Parmi les approches non médicamenteuses, la thérapie de groupe était plus efficace que le self help (RR = $1,98$; IC $95 \%: 1,20-2,46 ; n=13)$. De plus, l'efficacité de la semaine motivationnelle semble se confirmer à long terme ( 2 ans) alors que la population incluse présentait des facteurs défavorables à la réussite de l'arrêt. En effet, une forte dépendance, une comorbidité psychiatrique, une co-assuétude, un niveau élevé de stress et un environnement fumeur sont associés à des taux plus faibles d'abstinence alors que la motivation et un environnement non fumeur sont associés à des taux plus élevés [11]. Malgré la motivation importante à l'arrêt, la population incluse dans la semaine motivationnelle cumulait plusieurs facteurs défavorables : la forte dépendance physique, la dépression et la dépendance à l'alcool.

Parmi tous les facteurs étudiés, seuls la dépression ou des antécédents de dépression ont été identifiés comme des facteurs défavorables de l'arrêt. En comparaison avec la population générale, les personnes souffrant d'un trouble psychique ont effectivement deux fois plus de risque de fumer [13]. Plus spécifiquement, la dépression est plus fréquente chez les fumeurs [14]. Chez ces patients, les taux d'abstinence sont plus faibles et les taux de rechute plus élevés. La morbidité et la mortalité liées au tabac de la population atteinte de dépression sont donc plus importantes. Selon Lawrence et al., être atteint d'un trouble psychique comme la dépression est associé plus fréquemment au tabagisme régulier (OR :2,43; IC $95 \%: 1,97-3,01)$ et réduit les chances d'arrêt (OR : 0,77 ; IC $95 \%$ : 0,65-0,91) [15]. Le tabagisme et la dépression sont associés en raison de facteurs génétiques, biologiques et environnementaux communs $[15,16]$. La prise en charge du patient fumeur dépressif ou avec des antécédents de dépression n'est pas encore clairement établie. Une approche psychologique basée sur la confiance en soi, la motivation et l'amélioration de l'humeur semble plus efficace dans cette population [12, 16,17]. En effet, associer l'approche psychologique spécifique de l'humeur à l'approche standard du fumeur est plus efficace que l'approche standard seule chez le fumeur dépressif (RR : 1,47 ; IC $95 \%: 1,13$ 1,$92 ; n=11$ ) ou avec des antécédents de dépression (RR : 1,41; IC $95 \%: 1,13-1,77 ; n=13$ ). Mais, l'approche non médicamenteuse sans traitement pharmacologique est probablement insuffisante [16,17]. Associer une approche non médicamenteuse comme la semaine motivationnelle à une approche pharmacologique pourrait améliorer les chances d'abstinence de cette population. À l'inverse, seule la motivation évaluée par l'échelle analogique a été identifiée comme un facteur favorable de l'arrêt et confirme les données de la littérature [11]. Mais aucune différence significative n'a été retrouvée à long terme, probablement parce que la motivation est considérée comme un facteur important de l'initiation de l'arrêt plutôt que de la maintenance [18].

Enfin, même si la semaine motivationnelle semble efficace à long terme, les taux d'abstinence diminuent avec le temps. En effet, même si les différents traitements à notre disposition permettent d'améliorer de manière significative les taux d'abstinence, le sevrage tabagique est 
aussi associé à un taux élevé de rechutes lié aux caractéristiques particulières de la dépendance tabagique. Peu d'études ont été publiées à propos des taux de rechutes des différentes approches thérapeutiques à long terme. Agboola et al. [19] ont mis en évidence dans sa métaanalyse que parmi les approches non médicamenteuses, seul le self help était efficace dans la prévention des rechutes, entre 12 et 18 mois, chez les patients qui ont arrêté de fumer sans aide initialement (OR : 1,52; IC $95 \%: 1,15-2,01 ; n=3$ ). Parmi les approches médicamenteuses à long terme, le bupropion était efficace (OR : 1,49 ; IC $95 \%: 1,10-2,01 ; n=4$ ) ainsi que les substituts nicotiniques (OR : 1,33 ; IC $95 \%: 1,08-1,63 ; n=4$ ). Associer l'approche non médicamenteuse à un traitement médicamenteux est probablement la stratégie à adopter pour obtenir les meilleurs résultats d'abstinence à long terme.

Enfin, cette étude présente certaines limites puisqu'elle est rétrospective et qu'elle est basée uniquement sur les déclarations des patients, sans mesure objective d'arrêt du tabagisme (mesure du monoxyde et/ou de la cotinine urinaire).

En conclusion, la semaine motivationnelle semble être une approche efficace à long terme qui pourrait être utilisée dans le sevrage tabagique et proposée au patient motivé et favorable à une approche en groupe. Cette étude confirme que la dépression est un facteur défavorable de l'arrêt. De nouvelles stratégies comme associer la semaine motivationnelle à une approche médicamenteuse devraient être développées pour cette population à risque.

\begin{tabular}{|c|c|c|c|c|c|c|c|}
\hline & Variable/facteur & OR & IC $95 \%$ & $p$ & $\mathrm{ORa}$ & $1 C 95 \%$ & $p$ \\
\hline A 6 mois & $\begin{array}{l}\text { Dépression ou ATCD } \\
\text { Test de Fagerström } \\
\text { Test de Richmond } \\
\text { Motivation, échelle } \geq 8\end{array}$ & $\begin{array}{l}0,37 \\
0,60 \\
1,20 \\
2,57\end{array}$ & $\begin{array}{l}0,18-0,73 \\
0,29-1,24 \\
0,61-1,37 \\
1,35-4,89\end{array}$ & $\begin{array}{l}0,004 \\
0,163 \\
0,587 \\
0,004\end{array}$ & $\begin{array}{l}0,32 \\
0,60 \\
0,68 \\
3,28\end{array}$ & $\begin{array}{l}0,16-0,76 \\
0,26-1,40 \\
0,28-1,62 \\
1,45-7,40\end{array}$ & $\begin{array}{l}0,003 \\
0,239 \\
0,383 \\
0,004\end{array}$ \\
\hline A 1 an & $\begin{array}{l}\text { Dépression ou ATCD } \\
\text { Test de Fagerström } \\
\text { Test de Richmond } \\
\text { Motivation, échelle } \geq 8\end{array}$ & $\begin{array}{l}0,41 \\
0,42 \\
1,58 \\
2,21\end{array}$ & $\begin{array}{l}0,20-0,84 \\
0,20-0,87 \\
0,79-3,16 \\
1,12-4,34\end{array}$ & $\begin{array}{l}0,013 \\
0,020 \\
0,195 \\
0,026\end{array}$ & $\begin{array}{l}0,35 \\
0,46 \\
0,99 \\
2,47\end{array}$ & $\begin{array}{l}0,16-0,77 \\
0,20-1,06 \\
0,04-2,33 \\
1,08-5,64\end{array}$ & $\begin{array}{l}0,009 \\
0,069 \\
0,994 \\
0,033\end{array}$ \\
\hline$\AA 2$ ans & $\begin{array}{l}\text { Dépression ou ATCD } \\
\text { Test de Fagerström } \\
\text { Test de Richmond } \\
\text { Motivation, échelle } \geq 8\end{array}$ & $\begin{array}{l}0,25 \\
0,41 \\
1,80 \\
2,29\end{array}$ & $\begin{array}{l}0,10-0,58 \\
0,19-0,87 \\
0,97-3,73 \\
1,08-4,88\end{array}$ & $\begin{array}{l}0,001 \\
0,022 \\
0,116 \\
0,026\end{array}$ & $\begin{array}{l}0,27 \\
0,53 \\
1,20 \\
2,32\end{array}$ & $\begin{array}{l}0,11-0,65 \\
0,22-1,26 \\
0,50-2,92 \\
0,94-5,72\end{array}$ & $\begin{array}{l}0,004 \\
0,153 \\
0,685 \\
0,066\end{array}$ \\
\hline
\end{tabular}

\section{Déclaration de liens d'intérêts}

Les auteurs déclarent ne pas avoir de liens d'intérêts. 


\section{Annexe 1. Programme de la semaine motivationnelle}

\begin{tabular}{|c|c|c|}
\hline $\begin{array}{l}\text { Projet Re-Naissance } \\
\text { pour renaître de ses cendres } \\
\text { Programme d'aide à la } \\
\text { cessation tabagique } \\
\text { Centre d'Aide aux Fumeurs du } \\
\text { CHU-Brugmann }\end{array}$ & $\begin{array}{l}\text { OBJECTYF } \\
\text { Evaluer votre état de santé } \\
\text { biologique, psychologique et social } \\
\text { pour que vous établissiez une } \\
\text { stratégie qui rendra confortable } \\
\text { I'arrêt du tabac à long terme } \\
\text { INTERVENANTS } \\
\text { Une équipe pluridisciplinaire } \\
\text { intègre l'approche médicale et } \\
\text { psychologique du projet } \\
\text { M. De Baets : Tabacologue et } \\
\text { Coordlnatrice } \\
\text { Dr V. Lustygler : Psychlatre et } \\
\text { Tabacologue } \\
\text { Dr J. Thimpont : Pneumologue et } \\
\text { Tabacologue } \\
\text { Or S. Abdel Kafl : Pneumologue et } \\
\text { Tabacologue } \\
\text { Dr S. Gozlan: Psychlatre } \\
\text { C. Steenwinckel: Dlététlque } \\
\text { J.L. Carplaux : Sophrologue } \\
\text { L.M. Gourmet : Sophrologue } \\
\text { M. Ansseau : Psychologue } \\
\text { A. Van Malder : Hypnose } \\
\text { S. Minet : Psychothéraple } \\
\text { survy } \\
\text { Proposition de suivi en groupe } \\
\text { tous les premiers jeudi du mois } \\
\text { de 13h à 15h, pendant } 12 \text { mois }\end{array}$ & 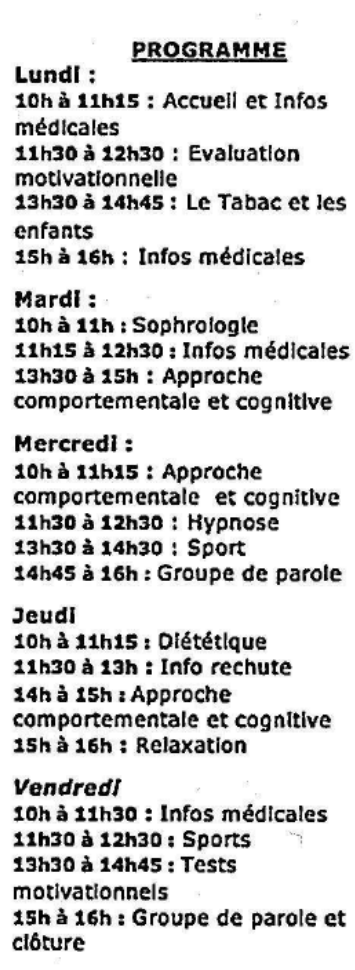 \\
\hline
\end{tabular}




\section{Références}

[1] Taylor DH, Hasselblad V, Henley SJ, et al. Benefits of smoking cessation for longevity. Am J Public Health 2002;92:990-6.

[2] Peto R, Darby S, Deo H, et al. Smoking, smoking cessation, and lung cancer in the UK since 1950: combination of national statistics with two case-control studies. BMJ 2000;321:323-9.

[3] Critchley JA, Capewell S. Mortality risk reduction with smoking cessation in patients with coronary heart disease: a systematic review. JAMA 2003;290:86-97.

[4] Center for Disease control and Prevention. Reducing tobacco use: a report of the surgeon general. Atlanta: U.S. Department of Health \& Human Services; 2000. www.cdc.gov/tobacco/data_statistics/sgr/2000/index.htm.

[5] Hughes JR, Keely J, Naud S. Shape of the relapse cue and long-term abstinence among untreated smokers. Addiction 2004;99:29-38.

[6] Ewing JA. Detecting alcoholism. The CAGE questionnaire. JAMA 1984;252:1905-7.

[7] Heatherton TF, Kozlowski LT, Frecker RC, et al. The Fagerstrèim Test for Nicotine Dependence: a revision of the Fagerstrèim Tolerance Questionnaire. Br J Addict 1991;86:1119-27.

[8] Richmond RL, Kehoe LA, Webster IW. Multivariate models for predicting abstention following intervention to stop smoking by general practioners. Addiction 1993;88:1127-35.

[9] Zigmond AS, Snaith RP. The hospital anxiety and depression scale. Acta Psychiatr Scand 1983;67:36170.

[10] Agboola SA, Coleman T, McNeiU A, et al. Abstinence and relapse among smokers who use varenicline in a quit attempt- a pooled analysis of randomized controlled trials. Addiction 2015;110: 1182-93.

[11] U.S. Department of Health and Human Services. Treating tobacco use and dependence: 2008 update; 2008.

http://www.ahrq.gov/professionals/cliniciansproviders/guidelines-recommendations/tobacco/index. html.

[12] Hartmann-Boyce J, Stead LF, Cahill K, et al. Efficacy of interventions to combat tobacco addiction: Cochrane update of 2013 reviews. Addiction 2014;109:1414-25.

[13] Lasser K, Boyd JW, Woolhandler S, et al. Smoking and mental illness: a population-based prevalence study. JAMA 2000;284:2606-10.

[14] Fergusson DM, Goodwin RD, Horwood LJ. Major depression and cigarette smoking: results of a 21year longitudinal study. Psychol Med 2003;33:1357-67.

[15] Lawrence D, Hafekost J, Hull P, et al. Smoking, mental illness and socioeconomic disadvantage: analysis of the Australian National Survey of Mental Health and Wellbeing. BMC Public Health 2013; 13:462.

[16] Weinberger AH, Mazure CM, Morlett A, et al. Two decades of smoking cessation treatment research on smokers with depres- sion: 1990-2010. Nicotine Tob Res 2013;15:1014-31.

[17] van der Meer RM, Willemsen MC, Smit F, et al. Smoking cessation interventions for smokers with current or past depression. Cochrane Database Syst Rev 2013;8:CD006102. 
[18] Borland R, Yong H, Balmford J, et al. Motivational factors predict quit attempts but not maintenance of smoking cessation: findings from the International Tobacco Control Four country project. Nicotine Tob Res 2010;12:S4-11.

[19] Agboola S, McNeill A, Coleman T, et al. A systematic review of the effectiveness of smoking relapse prevention interventions for abstinent smokers. Addiction 2010;105:1362-80. 